\title{
Producción y Comercialización de Productos del Chañar (Geoffroea decorticans (Gillies ex Hook. \& Arn.) Burkart) por descendientes de pueblos originarios de la Comuna de Copiapó. Región de Atacama. Chile
}

\author{
Production and marketing of Chañar products (Geoffroea Decorticans \\ (Gillies ex Hook. \& Arn.) Burkart) by descendants of native peoples \\ of the commune of Copiapó. Atacama region. Chile
}

\author{
María del Carmen Varela*1, Marlene González², Enrique Villalobos ${ }^{3}$, \\ Sandra Gacitúa*3, Jaime Montenegro ${ }^{3}$
}

\begin{abstract}
RESUMEN
Chañar (Geoffroea decorticans), es un árbol resistente a la sequía de zonas áridas y según varios autores ha sido parte importante de la dieta de pueblos como Atacameños, Collas, Diaguitas y Aimaras. Harina, arrope y derivados, se vinculan a la alimentación histórica de estas comunidades por su valor nutritivo, dado por altos contenidos de azúcares, proteínas y aceites, junto al reconocimiento de propiedades medicinales que aún se destacan para el alivio, principalmente de malestares respiratorios. En la actualidad, la producción artesanal y comercialización local de algunos de estos productos se realiza de manera informal, por lo tanto, se considera de importancia valorar el patrimonio tradicional del chañar y de los productos derivados de sus frutos. El interés manifestado por las actuales comunidades indígenas es mejorar las prácticas tradicionales y establecer un sello propio, para la especie y para los productos derivados del fruto. Para cumplir lo anterior, se realiza un estudio de caso en las comunidades Piedra Colgada y San Pedro, ambas ubicadas en la comuna de Copiapó, Región de Atacama. Se aplican métodos cualitativos y técnicas sociodemográficas para obtener información detallada de percepciones y experiencias que permitan establecer fortalezas, potencialidades, como así mismo, identificar riesgos y vulnerabilidades para enfrentar una eventual apertura de mercado. Este artículo, presenta consideraciones socioculturales acerca de la valorización de la producción y comercialización de los productos derivados de los frutos del chañar, que cumplen con la valoración sociocultural del rescate patrimonial y se formalice un nuevo escenario de comercialización, que beneficie a las comunidades asociadas que producen y comercializan los citados productos.
\end{abstract}

Palabras claves: Valor Patrimonial, Comunidades Indígenas, Arrope de Chañar, Harina de Chañar.

\begin{abstract}
Chañar (Geoffroea decorticans), is a tree resistant to the drought in arid zones and according to several authors it has been an important part of the diet of towns such as Atacameños, Collas, Diaguitas and Aimaras. Flour, syrup (arrope) and by-products, are linked to the historical feeding of these communities for their nutritional value, given by high content of sugar, proteins and oils, along with the recognition of medicinal properties that still stand out for the relief, mainly of respiratory ailments.

At present, the handmade production and local commercialization of some of these products is made in an informal way, therefore, it is considered of importance to value the traditional heritage of the chañar and the by-products got from its fruit.

The interest shown by the current indigenous communities is to improve traditional practices and put their own stamp, for the species and by-products from the fruit. To accomplish the above, a case study is carried out in the Piedra Colgada and San Pedro communities, both located in the district of Copiapó, Atacama Region.

Qualitative methods and sociodemographic techniques are applied to obtain detailed information on perceptions and experiences that allow to establish strengths, potentialities, as well as identify risks and vulnerabilities to face an eventual market opening. This article presents sociocultural considerations about the valorization of the production and commercialization of by-products from the fruit of the chañar, which comply with the sociocultural valuation of the heritage rescue and formalize a new marketing scenario, that benefits the associated communities that produce and market the aforementioned products.
\end{abstract}

Keywords: Heritage Value, Indigenous Communities, Chañar sypup (arrope), Chañar flour.

\footnotetext{
Universidad de La Serena. La Serena, Chile.

Instituto Forestal. Santiago, Chile.

Instituto Forestal sede Diaguitas. La Serena, Chile

* Autor por correspondencia: sgacitua@infor.cl
}

Fecha de Recepción: 10 de diciembre, 2018.

Fecha de Aceptación: 31 Agosto, 2019. 


\section{Introducción y revisión Bibliográfica}

Esta investigación se contextualiza en la valorización del rescate patrimonial de productos ancestrales agroalimentarios, por parte de las comunidades indígenas que poseen una tradición en recolección, producción y comercialización de estos, en este caso, derivados del chañar (Geoffroea decorticans). (Ver Figura 1).

Larraín (2011), señala que el fruto del chañar y sus derivados (arrope y harina) tuvieron una gran importancia en los viajeros que atravesaban el desierto de Atacama entre los siglos XIX y $\mathrm{XX}$; porque suministraban abundante cantidad de azúcares a través de su pulpa carnosa. El mismo autor, señala que en 1787 en la Zona de Atacama existían grandes bosques de chañares, los cuales eran protegidos por lo interesante de su fruto, a partir del cual elaboraban una bebida llamada "Quilapanada", consumida por los habitantes de esa época durante la celebración de sus fiestas; de igual manera, el fruto era utilizado como alimento para animales. La importancia de la existencia de chañares y de los atributos alimenticios y medicinales de sus productos se encuentran documentadas en las crónicas de historiadores del siglo XVI, como son las obras de Mariño de Lobera y Gerónimo de Vivar y en fuentes más contemporáneas en Latcham (1936); Hidalgo $(1989 ; 1972)$ y Gleisner y Montt (2014).

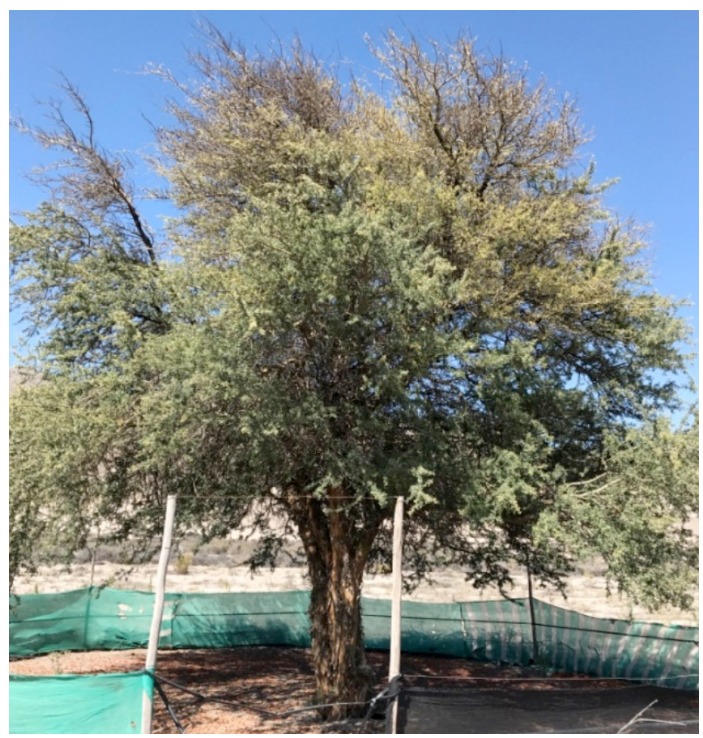

Figura 1. Imagen Chañar; Piedra Colgada. Valle de Copiapó. (Griem, 2002). Fuente: INFOR 2017.
Los cultivadores han construido una herencia e identidad en torno al chañar, que se manifiesta fundamentalmente de manera colectiva en las familias mediante celebraciones de cosmovisiones ancestrales y en momentos festivos de índole social, como la venida de un hijo desde la ciudad, para un cumpleaños, etc. En este marco, además se elaboraban una serie de postres, pan dulce, galletas, flanes, y otras preparaciones dulces a partir de su harina o de su arrope, los que se preparan para ocasiones especiales, como una forma de celebrar y disfrutar junto a los parientes o amigos. En relación a lo señalado, se cita a Contreras, 1992 textualmente: "Entendemos por sistema alimenticio el conjunto de representaciones, creencias y de prácticas heredadas y/o aprendidas que están asociadas a la alimentación y que son compartidas por los individuos de una cultura dada o de un grupo social determinado dentro de una cultura".

Por otra parte, es preciso señalar que en el ámbito económico y social la valorización del arrope y harina de chañar como PFNM (Productos Forestales No Madereros) y su proceso de elaboración, se vinculan directamente a la pequeña agricultura dado la fuerte dependencia de las comunidades indígenas para su subsistencia y complemento de ingresos, así mismo, como las personas involucradas en las tareas de recolección, procesamiento, producción y comercialización de productos señalados posibilitan el trabajo de hombres, mujeres, niños y ancianos, en el medio rural principalmente y en épocas de reducción de otras actividades (INFOR, 2017).

El estudio de caso de esta investigación de valoración patrimonial del chañar se centra en las comunidades indígenas, mayormente pertenecientes a la etnia colla, localizadas en Piedra Colgada y San Pedro, Comuna de Copiapó, Región de Atacama (Ver Figura 2). Estos asentamientos se insertan en medio de uno de los bosques mejor conservados de Chañar, principalmente en Piedra Colgada (Comunidad Piedra Luna); la mayoría de los miembros de estas comunidades son campesinos y desarrollan actividades agrícolas y pecuarias en la zona. Actualmente ellos administran bosques de chañar, los cuales no han sido manejados adecuadamente, para aumentar su producción y hacer de este recurso un aporte a su economía en forma sustentable.

Por lo citado anteriormente, el Proyecto FIA en desarrollo "Rescate patrimonial y puesta en 


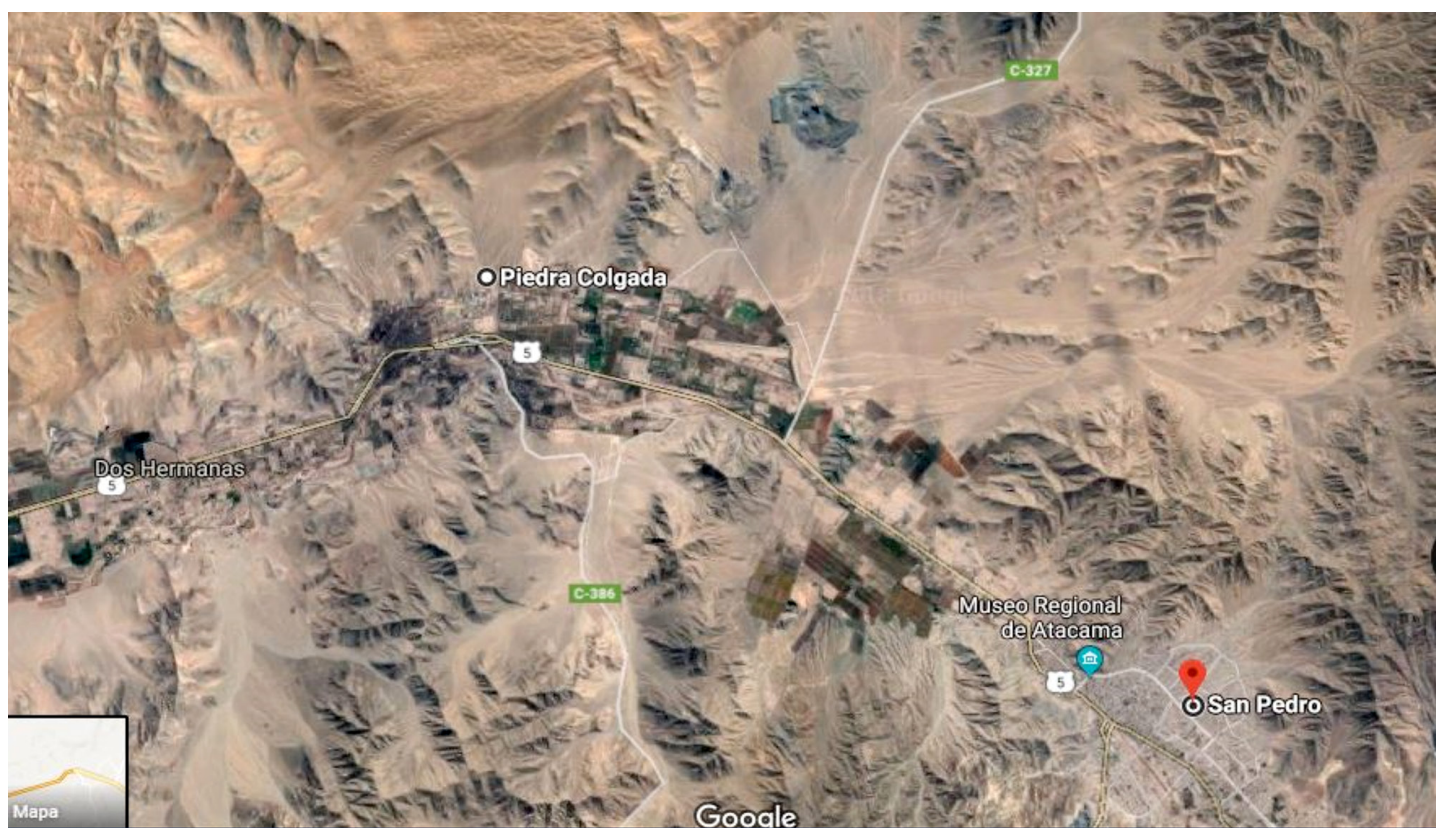

Figura 2. Localización de Comunidades Piedra Colgada y San Pedro. Comuna de Copiapó. Región Atacama. Fuente: Imagen satelital Google Maps. 2018.

valor de la producción Artesanal de Arrope y Harina derivados del fruto de Chañar, en la localidad de San Pedro y Piedra Colgada, comuna de Copiapó, Región de Atacama" presenta como objetivo general la caracterización del recurso actual del Chañar en términos de productividad frutal, con el fin de estimar y dimensionar el abastecimiento de materia prima potencial para la producción y comercialización de arrope y harina, y a su vez proyectar la sustentabilidad de potenciales aperturas de mercado para las localidades en estudio, permitiendo mejorar y/o completar los ingresos de las familias y comunidades indígenas.

El objetivo del presente artículo derivado del citado proyecto, es manifestar aspectos socioculturales de la valoración de la producción y la comercialización informal y de carácter ancestral, que hoy realizan las comunidades de origen indígena del lugar, para los productos derivados del chañar.

\section{Materiales y Métodos}

Para rescatar el conocimiento tradicional referido al uso y proceso productivo del Arrope y Harina de Chañar se utilizan procedimientos metodológicos que se enmarcan en estrategias y técnicas principalmente cualitativas, según metodologías descritas por Hernández, 2010 y Taylor \& Bodgan, 2012.

Específicamente, se inicia el estudio con una recopilación y revisión bibliográfica para identificar el origen y la evolución de la elaboración de arrope y harina de chañar a nivel local y regional; como así, para comprender la forma como se ha transmitido el saber-hacer asociado a la producción de los citados productos, a través de generaciones en las familias recolectoras-productoras.

En las fases metodológicas siguientes se aplican para el logro de los objetivos pretendidos:

- Elaboración de matriz de Localización Geográfica con datos geo-demográficos, y socioeconómicos, entre otros, de los integrantes de las comunidades.

- Entrevistas semi-estructuradas a productores y consumidores habituales de ambas localidades, con preguntas relacionadas a percepciones y experiencias en el proceso de producción y comercialización actuales, del pasado heredado y expectativas futuras.

- Entrevistas semi-estructuradas a referentes externos principalmente profesionales vinculados al tema (INDAP; CONAF; INACAP; Universidades regionales y otros) 
- Actividad Práctica en Terreno: Elaboraciones de Arrope y Harina por los miembros de las comunidades en estudio. Material Base: Frutos del chañar de tres localidades geográficas locales, ubicadas en sectores de costa, valle medio y precordillerano de la comuna.

- Talleres de Información, discusión y difusión de resultados de aplicaciones anteriores, previamente procesadas con métodos cualitativos y complementos cuantitativos, según autores citados.

\section{Resultados y Discusión}

Estimando los procedimientos metodológicos y técnicas sociodemográficas utilizadas se establecen en primer lugar, consideraciones socioculturales que mayoritariamente son consensuadas por los participantes de las dos comunidades de origen indígena: Piedra Luna con asentamiento en Piedra Colgada y Serranía Poblete en San Pedro de Copiapó. Si bien los componentes de Piedra Luna reconocen ser descendientes del pueblo Colla, los pobladores del segundo citado, estiman que desde el punto de vista genealógico sus pobladores proceden de diferentes troncos sanguíneos, entre los cuales se encuentran igualmente collas, diaguitas y mapuches, pueblos reconocidos y validados jurídicamente (Ley Indígena $\mathrm{N}^{\circ} 19593$, 1993.CONADI). No obstante ello, el contacto de ambas comunidades con vecinos y familiares locales, sensibilizan el sentir que el Chañar es un recurso local patrimonial, el cual se debe preservar, como así, la producción del fruto en productos y subproductos. Socialmente, el vínculo matrimonial o de convivencia entre personas de origen colla local con otras etnias, constituye un fuerte factor de unión y difusión de la elaboración del arrope y otros productos a partir del chañar, que no distan de los realizados en el pasado. En relación al procesamiento del arrope, que ocupa varias horas (promedio 08 horas) señalan que la recolección del chañar y la producción de arrope y otros, se inicia en los sectores más altos hacia la cordillera de la comuna, especialmente familias oriundas de la localidad de Manfla. La tradición asimilada, manifiesta que en todo tiempo han sido recolectores del fruto y las actividades se han desarrollado en los grupos familiares; en la actualidad la generación de más años bordea los 80 (emisores de la tradición) y los involucrados en las actividades vinculadas al recurso, es decir, receptores de la tradición, promedia los 46 años. Antiguamente niños y adolescentes se vinculaban a la recolección de frutos y disfrutaban de participar del procesamiento de los productos; hoy son más lejanos, por razones de estudio y/o trabajo en lugares de vida más distanciados y relacionado también con el deseo de sus padres de mejorar la calidad de vida. En estos días, las mujeres han tomado un rol más preponderante y se han agrupado en asociaciones como RATMURI (Red de Atacama de Mujeres Rurales e Indígenas). El quehacer de estas mujeres se vincula a prácticas gastronómicas y artesanales heredadas de madres y abuelas. En segundo término, en relación a la producción y comercialización es importante destacar, la realización de Talleres participantes con la concurrencia de miembros de ambas comunidades, para consensuar testimonios y experiencias señaladas como resultados del procesamiento de entrevistas y otras técnicas metodológicas aplicadas. A modo de ejemplo como discusión, se presentan algunos aspectos específicos en el proceso de la elaboración de arrope. Se prioriza la necesidad de conciliar diferencias detectadas en las etapas del proceso y en algunos detalles específicos para cada una de ellas, como tipo de recolección, volumen de frutos seleccionados, cantidad de agua en la elaboración, utensilios utilizados, tiempo ocupado para cada etapa, fuente de energía ocupada, envases requeridos, entre otros.

A partir de algunas preguntas preestablecidas, por parte del equipo investigador INFOR para cada etapa, se fue analizando y revisando cada una de ellas, estableciendo acuerdos fundamentados y detectando si faltaban pasos intermedios importantes de destacar o corrigiendo el lenguaje común a utilizar y confirmando la información entregada por la comunidad en actividades anteriores y testimonios de referentes con experiencia en el tema.

A modo de síntesis, se entrega en el diagrama siguiente (Figura 3) las diversas etapas del proceso de elaboración del arrope, validadas por productores y referentes especialistas; estas se estiman como adecuadas para las buenas prácticas de la producción de Arrope, sin desconocer los atributos naturales del fruto.

De igual manera que el procedimiento señalado, se consensuó el proceso de elaboración de harina, destacando que son los menos quienes 


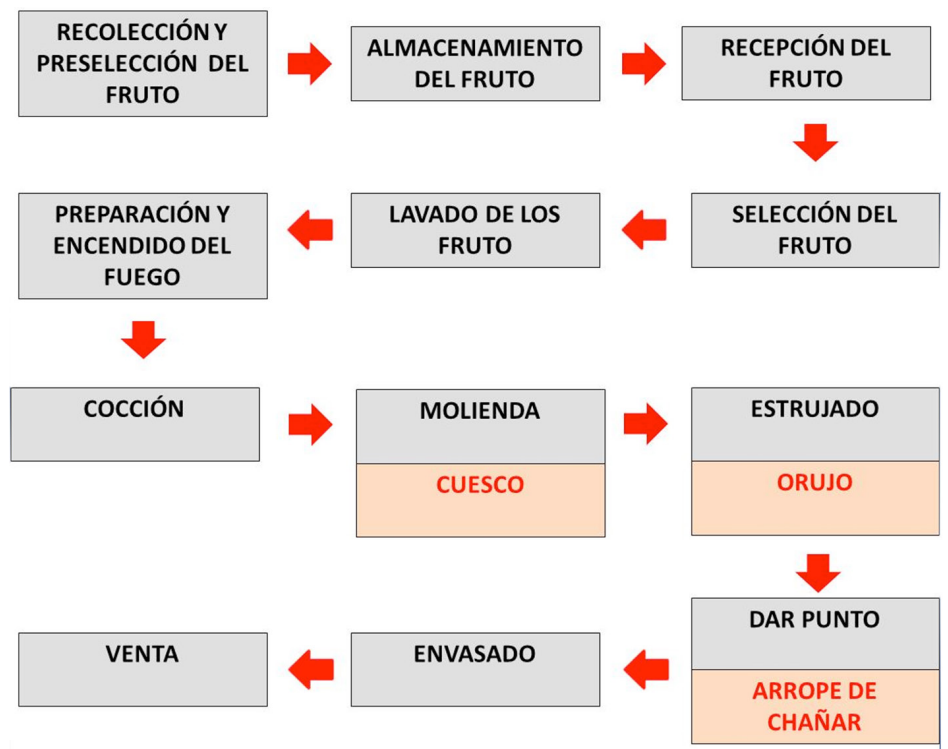

Figura 3. Diagrama del proceso de elaboración de Arrope de Chañar.

Fuente: Taller INFOR - Gobierno Regional Copiapó. 2018. Región de Atacama.

elaboran este producto, aunque la importancia de la harina de chañar ha sido vital en el pasado como alimento mezclado con leche (cocho), básico para crianceros de las partes más altas de la comuna. En la actualidad de la harina, se derivan una serie se subproductos especialmente de utilización en Pastelería, como galletas y panecitos, agradables al gusto del consumidor.

En relación a la comercialización de los productos, la discusión se centra en algunos puntos, aunque existe la opinión unánime que el tiempo ocupado para la elaboración de estos productos, no es compensada, por el valor que se obtiene de la comercialización.

Por otra parte, se reconoce la informalidad de este comercio, poca claridad en cuanto a los valores de los productos en el mercado formal; coexisten además otras comunidades de localizaciones vecinas al sector de origen colla u otra etnia, que son cultivadoras y/o compradoras de frutos y productoras de arrope, harina que entran a competir en el mercado informal, existiendo también venta del producto en el mercado formal por productores no vinculados a la etnia colla. La forma normal de establecer las transacciones comerciales son las conexiones con familiares y vecinos cercanos, a veces, se viaja a otras ciudades como Antofagasta por el norte y La Serena por el sur, aprovechando el viaje para visitar parientes y amigos. En ocasiones, la posibilidad de manejo de internet por algún miembro de la familia, sirve para extender la venta a ciudades y pueblos más distantes.

En relación a los lugares de venta se sigue la línea tradicional: sitios de acceso público como son centros de salud (por su reconocida propiedad medicinal del arrope) y otros servicios públicos como correos, terminal de buses, principalmente. Un centro importante de venta es la plaza de Copiapó, en la cual se establecen algunos puestos ocasionales, principalmente en periodos de mayor turismo en la zona (temporada veraniega y manifestación territorial del Desierto Florido).

La informalidad de la producción y comercialización de estos productos conlleva a ciertas potencialidades que los productores involucrados reconocen deben ser resueltos, por ejemplo el interés de mantener los bosques de Chañar mediante capacitaciones de instituciones como INFOR u otras que entreguen las herramientas necesarias para cumplir con este requerimiento. De igual modo, aprecian la entrega de prácticas mejoradas en los procesos de elaboración del arrope, harina y subproductos. Para ambas comunidades, el valor del atributo gastronómico y medicinal es innegable y lo ha sido a través de los tiempos ancestrales y en la actualidad para el chañar y sus productos, debe generarse medidas tendientes a lograr una apertura de mercado más formal, probablemente logrando una mejor organización de los participantes. 
Sin embargo, se reconoce paralelamente algunas debilidades vinculadas a competencias y rivalidades geoespaciales entre grupos urbanos y rurales; los costeros y de cordillera; y la de los más antiguos de procedencia indígena anterior a la Ley CONADI y los reconocidos con posterioridad. En este ámbito, la discusión no solo se centra por rivalidades en el mercado de los productos, sino en problemas relacionados con la asignación transitoria de tenencia de la tierra, contradiciendo el deseo de la posesión permanente; otra demanda generalizada es tener acceso a una mejor disponibilidad del recurso agua; para la comunidad Piedra Luna es más importante su acceso para el desarrollo de las actividades (también de orden silvícola), que para los de Serranía Poblete, que por ubicación geográfica más cercana al río tienen mejor disponibilidad al recurso. No obstante, esta cercanía al río constituye un mayor riesgo y vulnerabilidad socio ambiental; los aluviones de los años 2015 y 2017 ocasionados por lluvias anormales causaron grandes pérdidas de bienes materiales como de individuos de chañar emplazados en terrenos de la comunidad.

Por último, es importante destacar como factor de potencialidad para escenarios futuros de apertura a un mercado formal, el emplazamiento de las comunidades en estudio, estas se localizan en un territorio geográfico que si bien es de condición árida, esta no ha sido obstáculo para el crecimiento y permanencia natural de esta especie; muy favorable además, es la condición topográfica del terreno del asentamiento, ya que se ubican preferentemente en pendientes bajas, lo que facilita la conectividad y accesibilidad entre los lugares del entorno y otros más lejanos.

\section{Conclusiones}

Una conclusión básica y generalizada es que los productores en su mayoría recolectan el fruto, procesan los productos en sus hogares y en fogones preparan, por ejemplo, el arrope directamente junto al grupo familiar; de igual modo, se comercializa en el entorno conformado por parientes y vecinos utilizando envases de vidrio con volúmenes y precios de venta muy variables en el mercado informal local.

El estudio indica que si bien las comunidades indígenas con las que se ha trabajado en este territorio comprenden la valoración patrimonial que tiene la especie Geoffroea decorticans y pretenden rescatar lo trasmitido a través de generaciones en la elaboración de productos y subproductos a partir de su fruto, también reconocen la necesidad de aprender a reconocer el valor que tiene la especie en términos económicos, junto a la existencia de otros grupos nuevos que ingresan en la actualidad al mercado que se transforman en competidores, aun considerando las condición actual de informalidad de la comercialización.

Es interesante destacar que tradicionalmente las mujeres han jugado un rol preponderante en el procesamiento de los productos, comportamiento que en estos días es posible de visualizar en el proceso de elaboración del arrope y otros. Este comportamiento social de matriarcado es habitual en las comunidades indígenas en Chile, y ha sido reforzada con los testimonios de historiadores y antropólogos de la región.

Por otra parte, es necesario, así lo estiman investigadores y referentes externos, que los productores, deberían establecer mayores vínculos entre ellos, sugiriéndoles aunar esfuerzos y articulaciones entre sí; comprendiendo que todos forman parte de una cadena que sustentan objetivos comunes. Se sugiere estructurar una estrategia comercial, considerando estos aspectos para lograr una manera de formalizar una apertura de mercado. Una opción podría ser, reunir la producción de todos y comercializar sólo un producto uniforme, que sea representativo y que cuente con un sello de identidad local. Por otra parte, se potencia el escenario actual frente al surgimiento durante los últimos años de una nueva generación de alimentos procesados en el país, única, sofisticada, competitiva y con altos estándares de calidad; esta generación de alimentos está conformada por los productos conocidos como Gourmet, utilizados y promocionados por importantes chefs de la cocinería nacional e internacional; se estima que los atributos gastronómicos de los productos del chañar podrían perfectamente incorporarse en esta línea ante una eventual apertura de mercado.

Finalmente, señalar que la valoración de los productos del chañar en el mercado local generarían perspectivas de comercio hacia otras regiones del país, con beneficios económicos para las comunidades productoras involucradas y en general a los habitantes del territorio copiapino. Se vislumbra como un factor relevante implantar un sello de identidad y de recurso patrimonial del chañar en la Región de Atacama. 


\section{Literatura Citada}

Contreras, J.

1992. Alimentación y cultura: reflexiones desde la Antropología. Revista Chilena de Antropología. Santiago. Chile, 11: 95-11.

Gleisner, C., \& Montt, S.

2014. Serie introducción histórica y relatos de los Pueblos Originarios de Chile. Colla. Santiago de Chile: Unidad de Cultura FUCO. Proyecto FONDART. 104 pp.

Grass-Ramírez, J. Cervantes-Escoto, F; Palacios-Rangel, M. 2016. Elementos metodológicos para el fortalecimiento del enfoque de sistemas agroalimentarios localizados (SIAL). Agricultura, sociedad y desarrollo, 13(1): 63-85.

Hernández, S.

2010. Metodología de la Investigación. Ed. Mc Graw Hill Interamericana. México. 656 p.

Hidalgo, J.

1972. Culturas Protohistóricas del Norte de Chile. Cuadernos de Historia $N^{\circ} 1$, Departamento de Historia. Facultad de Filosofía y Educación, Universidad de Chile. Santiago. 98 p.

Hidalgo, J et al.

1989. Prehistoria. Desde sus orígenes hasta los albores de la conquista. Series Culturas de Chile Editorial Andrés Bello. Santiago. Chile. 460 pp.

Instituto Forestal (INFOR).

2017. Rescate del conocimiento tradicional del Patrimonio alimentario del Chañar y sus derivados. Informes Avance. Proyecto FIA "Rescate patrimonial y puesta en valor de la producción artesanal de arrope y harina derivados del fruto de Chañar, en la localidad de San Pedro y Piedra Colgada, comuna de Copiapó, Región de Atacama".

Larraín, $\mathrm{H}$.

2011. Chañar árbol frutal imprescindible para Atacameños y Aimaras: Testimonios históricos y experiencias. Disponible en: http://eco-antropologia.blogspot.cl/2011/08/el-chanararbol-frutal-de-atacamenos-y.html. Consultado: $16 \mathrm{de}$ abril.2016.

Latcham, R.

1936. La agricultura en Chile y en los países vecinos. Ediciones de la Universidad de Chile, Santiago. Chile. 336 pp.

Mariño de Lobera, P.

1970. Crónica del reino de Chile / escrita por Pedro Mariño de Lobera dirigida a García Hurtado de Mendoza; reducido a nuevo método y estilo por Bartolomé de Escobar; introducción y notas de Juan Uribe Echeverría. Santiago. Editorial Universitaria. Santiago. Chile. 358 p.

Ministerio de Planificación y Cooperación 1993. Ley Indígena No 19593 , CONADI. Corporación Nacional de Desarrollo Indígena (Chile).Santiago. Chile. 30 p. Taylor, J.; Bogdan, R.

2000. Introducción a los métodos cualitativos de Investigación. Ed. Paidós. México. 352 p.

Vivar G. Feliu C.

1966. Crónica y relación copiosa y verdadera de los reinos de Chile. Santiago. Instituto Geográfico Militar. 232 p. 


